\title{
On boundary correlators in Liouville theory on $\mathrm{AdS}_{2}$
}

\author{
Matteo Beccaria ${ }^{a}$ and Arkady A. Tseytlin ${ }^{b, 1}$ \\ ${ }^{a}$ Dipartimento di Matematica e Fisica Ennio De Giorgi, Università del Salento \& INFN, \\ Via Arnesano, 73100 Lecce, Italy \\ ${ }^{b}$ Blackett Laboratory, Imperial College, \\ Prince Consort Road, London, SW7 2BW, U.K. \\ E-mail: matteo.beccaria@le.infn.it, tseytlin@imperial.ac.uk
}

ABSTRACT: We consider the Liouville theory in fixed Euclidean $\mathrm{AdS}_{2}$ background. Expanded near the minimum of the potential the elementary field has mass squared 2 and (assuming the standard Dirichlet b.c.) corresponds to a dimension 2 operator at the boundary. We provide strong evidence for the conjecture that the boundary correlators of the Liouville field are the same as the correlators of the holomorphic stress tensor (or the Virasoro generator with the same central charge) on a half-plane or a disc restricted to the boundary. This relation was first observed at the leading semiclassical order (tree-level Witten diagrams in $\mathrm{AdS}_{2}$ ) in [19] and here we demonstrate its validity also at the one-loop level. We also discuss arguments that may lead to its general proof.

KeYwords: AdS-CFT Correspondence, Conformal Field Theory

ARXiv EPrint: 1904.12753

\footnotetext{
${ }^{1}$ Also at the Institute for Theoretical and Mathematical Physics, Moscow State University and Lebedev Institute, Moscow, Russia.
} 


\section{Contents}

1 Introduction 1

2 Liouville theory on Euclidean $\mathrm{AdS}_{2}$ background 6

$\begin{array}{lll}2.1 & \text { Two alternative formulations } & 7\end{array}$

2.2 One-point and two-point correlation functions 9

3 Three-point boundary correlation function $\quad 10$

4 Four-point boundary correlation function $\quad 14$

$\begin{array}{llr}\mathbf{A} \quad \mathrm{AdS}_{2} \text { integrals in disc parametrization } & \mathbf{1 8}\end{array}$

$\begin{array}{ll}\text { A.1 Tadpole reduction identity } & 18\end{array}$

$\begin{array}{lll}\text { A.2 One-loop correction to the two-point function } & 19\end{array}$

B On the one-point function in the ZZ and AdS formulations 21

C Tree level Witten diagram contributions to $\left\langle\Phi\left(t_{1}\right) \cdots \Phi\left(t_{4}\right)\right\rangle \quad 21$

D Global conformal block decomposition of the four-point correlator 22

\section{Introduction}

Study of quantum field theories in $\mathrm{AdS}_{2}$ background is of interest from several points of view (see, e.g., [1-6] and references there). A recent example appeared in the investigation of correlators of operators inserted on a straight or circular Wilson line in the context of $\mathrm{AdS}_{5} / \mathrm{CFT}_{4}$ correspondence [7-14]. As discussed in [12], starting with the string action in $\mathrm{AdS}_{5} \times S^{5}$ and expanding it near the corresponding minimal surface one gets a $2 \mathrm{~d}$ field theory action in $\mathrm{AdS}_{2}$ background. The boundary correlators of elementary excitations that should match the strong coupling limit of the Wilson loop "defect" correlators in $\mathcal{N}=4$ super Yang-Mills theory are constrained by the isometry of $\mathrm{AdS}_{2}$ or the $1 \mathrm{~d}$ conformal group $\mathrm{SO}(2,1) \simeq \mathrm{SL}(2, \mathbb{R})$ providing a non-trivial example of a non-gravitational $\mathrm{AdS}_{2} / \mathrm{CFT}_{1}$ duality. One technical problem in the approach of $[12,14]$ is how to systematically compute loop corrections to boundary correlators of $2 \mathrm{~d}$ quantum fields in $\mathrm{AdS}_{2}$.

Using this as a partial motivation, it is interesting to study loop corrections to boundary correlators in some simple 2d QFTs defined on $\mathrm{AdS}_{2}$ background. Here we shall consider the familiar example of the Liouville theory [15-17]

$$
S=\frac{1}{4 \pi} \int d^{2} x \sqrt{g}\left(\partial^{a} \varphi \partial_{a} \varphi+\mu e^{2 b \varphi}+Q R \varphi\right), \quad Q=b+b^{-1} .
$$


Defined on a fixed curved $2 \mathrm{~d}$ background it is a Weyl-covariant quantum theory with the central charge $c=1+6 Q^{2}$. In the special case of the Euclidean $\mathrm{AdS}_{2}$ background $d s^{2}=$ $\frac{1}{z^{2}}\left(d t^{2}+d z^{2}\right)$ with the curvature $R=-2$ the field $\varphi$ expanded near its constant vacuum value has the classical $(b \ll 1)$ mass $m^{2}=2$. Interpreting this model from the point of view of the $\mathrm{AdS}_{2} / \mathrm{CFT}_{1}$ duality ${ }^{1}$ and assuming the standard (Dirichlet) boundary condition at $z=0$ this field should have the boundary asymptotics $\left.\varphi(t, z)\right|_{z \rightarrow 0}=z^{2} \Phi(t)+\ldots$, i.e. should be dual to a $1 \mathrm{~d}$ boundary CFT operator with the $1 \mathrm{~d}$ conformal dimension $\Delta=2{ }^{2}$ One can then compute the corresponding boundary correlators ${ }^{3}$

$$
\left\langle\Phi\left(t_{1}\right) \cdots \Phi\left(t_{n}\right)\right\rangle \equiv \lim _{\mathrm{z}_{1}, \ldots, \mathrm{z}_{n} \rightarrow 0} \mathrm{z}_{1}^{-2} \cdots \mathrm{z}_{n}^{-2}\left\langle\varphi\left(t_{1}, z_{1}\right) \cdots \varphi\left(t_{n}, z_{n}\right)\right\rangle
$$

using the standard Witten diagram prescription.

Given the special conformal invariance properties of the Liouville theory one may expect these boundary correlators to have a particularly simple structure. Indeed, it was recently noticed in [19] that at the tree-level $(b \ll 1$ or $c \gg 1)$ the 2 -, 3- and 4-point boundary correlators (1.2) have exactly the same dependence on the boundary points $t_{i}$ as the correlators of the holomorphic stress tensor $T(z)$ (generator of the Virasoro algebra with the same central charge $c$ ) have on the complex coordinates $z_{i}$, i.e.

$$
\left\langle\Phi\left(t_{1}\right) \cdots \Phi\left(t_{n}\right)\right\rangle=\left.\kappa^{n}\left\langle T\left(z_{1}\right) \cdots T\left(z_{n}\right)\right\rangle\right|_{z_{i} \rightarrow t_{i}} .
$$

Here $\kappa=\kappa(b)$ is a proportionality coefficient in the formal identification $\Phi(t) \rightarrow \kappa T(t)$. Equivalently, one may view the r.h.s. of (1.3) as the correlator of the chiral stress tensor of a CFT on a half-plane (that may identified with $\mathrm{AdS}_{2}$ ) restricted to the real boundary $z_{i}=t_{i}+i z_{i} \rightarrow t_{i}$ (assuming the usual conformal gluing condition $T(t)=\bar{T}(t)$ ). Explicitly, the Virasoro algebra or the standard OPE, $T\left(z^{\prime}\right) T(z)=\frac{c / 2}{\left(z^{\prime}-z\right)^{4}}+\frac{2 T(z)}{\left(z^{\prime}-z\right)^{2}}+\frac{\partial_{z} T(z)}{z^{\prime}-z}+\ldots$, fixes the correlators of the stress tensor $T(z)$ to be

$$
\begin{aligned}
\left\langle T\left(z_{1}\right) T\left(z_{2}\right)\right\rangle= & \frac{c}{2 z_{12}^{4}}, \quad\left\langle T\left(z_{1}\right) T\left(z_{2}\right) T\left(z_{3}\right)\right\rangle=\frac{c}{z_{12}^{2} z_{13}^{2} z_{23}^{2}}, \\
\left\langle T\left(z_{1}\right) T\left(z_{2}\right) T\left(z_{3}\right) T\left(z_{4}\right)\right\rangle= & \frac{c^{2}}{4}\left(\frac{1}{z_{12}^{4} z_{34}^{4}}+\frac{1}{z_{13}^{4} z_{24}^{4}}+\frac{1}{z_{14}^{4} z_{23}^{4}}\right) \\
& +c\left(\frac{1}{z_{12}^{2} z_{23}^{2} z_{34}^{2} z_{14}^{2}}+\frac{1}{z_{13}^{2} z_{24}^{2} z_{14}^{2} z_{23}^{2}}+\frac{1}{z_{12}^{2} z_{24}^{2} z_{34}^{2} z_{13}^{2}}\right) .
\end{aligned}
$$

The fact that the boundary operator dual to the Liouville field $\varphi$ should have the dimension 2 is indeed consistent with the structure of $(1.4),(1.5) .^{4}$

Our aim will be to check the conjecture (1.3) beyond the leading tree level approximation discussed in [19] by directly computing the one-loop corrections to the correlators

\footnotetext{
${ }^{1}$ This approach is different in spirit from the earlier investigations in [2] and [5, 18].

${ }^{2}$ This is the special case of the familiar $\operatorname{AdS}_{d+1}$ relation $m^{2}=\Delta(\Delta-d)$.

${ }^{3}$ Here the l.h.s. may be viewed as a symbolic notation for the corresponding boundary CFT correlator.

${ }^{4} T$ has indeed dimension 2 with respect to the $\mathrm{SL}(2, \mathbb{R})$ subgroup of the $2 \mathrm{~d}$ conformal group. Note also that $\langle T\rangle=0$ by the conformal symmetry in the bulk so one needs to consider only the connected correlators or subtract the expectation value $\varphi \rightarrow \varphi-\langle\varphi\rangle$ in (1.2) to make the identification (1.3) possible.
} 
$\left\langle\Phi\left(t_{1}\right) \cdots \Phi\left(t_{n}\right)\right\rangle$ in (1.2) starting with the Liouville action (1.1) in $\mathrm{AdS}_{2}$. We shall also discuss how one may try to prove the relation (1.3) general and suggest the exact expression for $\kappa(b)$.

One prediction of the identification (1.3) is that the boundary operator dual to $\varphi$ should have no anomalous dimension, i.e. the two-point and three-point functions (1.2) should be given by

$$
\left\langle\Phi\left(t_{1}\right) \Phi\left(t_{2}\right)\right\rangle=\frac{C_{2}(b)}{t_{12}^{4}}, \quad\left\langle\Phi\left(t_{1}\right) \Phi\left(t_{2}\right) \Phi\left(t_{3}\right)\right\rangle=\frac{C_{3}(b)}{t_{12}^{2} t_{13}^{2} t_{23}^{2}},
$$

i.e. any logarithmic corrections should cancel out. The consistency with (1.3), (1.4) implies

$$
C_{2}=\frac{1}{2} c \kappa^{2}, \quad C_{3}=c \kappa^{3}, \quad \frac{\left(2 C_{2}\right)^{3}}{\left(C_{3}\right)^{2}}=c, \quad \frac{C_{3}}{2 C_{2}}=\kappa .
$$

Since $\kappa(b)$ is the only a priori unknown function (the central charge is assumed to be given by the Liouville theory value $\left.c=1+6\left(b+b^{-1}\right)^{2}=6 b^{-2}+\ldots\right)$ we thus get non-trivial relations between the coefficients in the perturbative expansion of the functions $C_{2}$ and $C_{3}$

$$
C_{2}(b)=C_{2,0}+C_{2,1} b^{2}+C_{2,2} b^{4}+\cdots, \quad C_{3}(b)=C_{3,0} b+C_{3,1} b^{3}+C_{3,2} b^{5}+\cdots,
$$

in particular,

$$
\text { (i) } \frac{4\left(C_{2,0}\right)^{3}}{\left(C_{3,0}\right)^{2}}=3, \quad \text { (ii) } \frac{8\left(C_{2,0}\right)^{2}}{\left(C_{3,0}\right)^{3}}\left(3 C_{2,1} C_{3,0}-2 C_{2,0} C_{3,1}\right)=13 .
$$

The tree-level relation (i) in (1.9) was checked in [19] and we will show that the one-loop relation (ii) is also satisfied.

It was also observed in [19] that the 4-point boundary correlator given by the sum of the disconnected and connected tree-level diagrams following from the Liouville action (1.1), i.e. symbolically

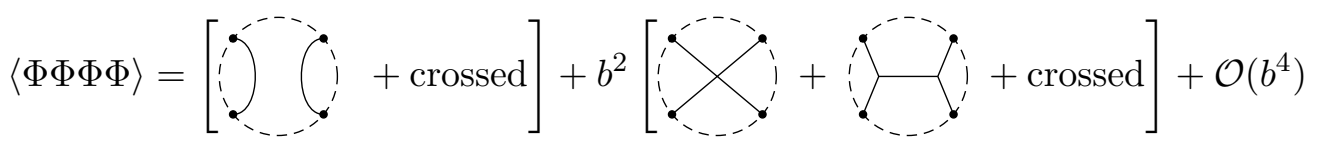

has a structure which is consistent with (1.3), (1.5). In general, one should expect to find

$$
\begin{aligned}
\left\langle\Phi\left(t_{1}\right) \cdots \Phi\left(t_{4}\right)\right\rangle & =\left\langle\Phi\left(t_{1}\right) \cdots \Phi\left(t_{4}\right)\right\rangle_{\text {disconn }}+\left\langle\Phi\left(t_{1}\right) \cdots \Phi\left(t_{4}\right)\right\rangle_{\text {conn }}, \\
\left\langle\Phi\left(t_{1}\right) \cdots \Phi\left(t_{4}\right)\right\rangle_{\text {disconn }} & =\left[C_{2}(b)\right]^{2}\left(\frac{1}{t_{12}^{2} t_{34}^{2}}+\frac{1}{t_{13}^{2} t_{24}^{2}}+\frac{1}{t_{14}^{2} t_{23}^{2}}\right), \\
\left\langle\Phi\left(t_{1}\right) \cdots \Phi\left(t_{4}\right)\right\rangle_{\text {conn }} & =C_{4}(b)\left(\frac{1}{t_{12}^{2} t_{23}^{2} t_{34}^{2} t_{14}^{2}}+\frac{1}{t_{13}^{2} t_{24}^{2} t_{14}^{2} t_{23}^{2}}+\frac{1}{t_{12}^{2} t_{24}^{2} t_{34}^{2} t_{13}^{2}}\right), \\
C_{4}=c \kappa^{4} & =C_{4,0} b^{2}+C_{4,1} b^{4}+C_{4,2} b^{6}+\cdots .
\end{aligned}
$$

Using (1.7), (1.9) to eliminate $\kappa$ we should then have the following relations for the coefficients in the perturbative expansion of $C_{4}$ in (1.14)
(iii) $C_{4,0}=\frac{\left(C_{3,0}\right)^{2}}{2 C_{2,0}}$,
(iv) $C_{4,1}=\frac{C_{3,0}}{2\left(C_{2,0}\right)^{2}}\left(2 C_{2,0} C_{3,1}-C_{2,1} C_{3,0}\right)$. 
The tree-level relation (iii) was checked in [19] while the non-trivial one-loop relation (iv) will be confirmed below. ${ }^{5}$

Our one-loop results will thus provide a strong evidence for the validity of the conjecture (1.3). One may wonder how it can be proved in general. The conformal symmetry of the Liouville theory in the bulk (on half-plane) viewed as an infinite global symmetry extending the $\mathrm{SL}(2, \mathbb{R})$ isometry of the $\mathrm{AdS}_{2}$ implies strong constraints on the boundary correlators which are effectively equivalent to the Virasoro symmetry constraints on the form of the stress tensor correlators (1.4), (1.5). This suggests that here we get an effective $\mathrm{CFT}_{1}$ which is simply a restriction of a $\mathrm{CFT}_{2}$ defined on a half-plane to its real-line boundary so that in this case the $\mathrm{AdS}_{2} / \mathrm{CFT}_{1}$ becomes effectively " $\mathrm{AdS}_{2} /\left(C F T_{2}\right)^{1 / 2}$ duality". ${ }^{6}$

In the semiclassical limit of small $b$ the relation (1.3) may then be argued for as follows. Since the theory (1.1) is Weyl-covariant theory in the bulk, in this limit we may eliminate the conformal factor of the $\mathrm{AdS}_{2}$ metric by redefining the Liouville field as $\varphi(t, z) \rightarrow$ $\phi(t, z)+b^{-1} \log z$, i.e. transform the action in (1.1) into the flat-space one (cf. $\left.[2,5]\right)$. This gives the Liouville CFT for field $\phi$ defined on a flat upper half-plane $(w=t+i \mathbf{z}, \mathbf{z}>0)$ with the (classical) stress tensor $T(w)=-\left(\partial_{w} \phi\right)^{2}+b^{-1} \partial_{w}^{2} \phi\left(\right.$ where $\partial_{w}=\frac{1}{2}\left(\partial_{t}-i \partial_{z}\right)$ ). The shifted field has then the boundary asymptotics $\left.\phi(t, \mathrm{z})\right|_{\mathrm{z} \rightarrow 0}=\mathrm{z}^{2} \Phi(t)-b^{-1} \log \mathrm{z}+\ldots$ Taking the boundary limit in $T(w \rightarrow t)$ gives

$$
T(t)=-\frac{3}{2} b^{-1} \Phi(t)+\mathcal{O}\left(z^{2}\right)
$$

This is precisely the operator relation which is required for the validity of (1.3) with $\kappa(b)=-\frac{2}{3} b+\cdots$. The same value of the leading term in $\kappa(b)$ in (1.3), (1.7) will be found by an explicit computation below (see (3.22)).

This argument should admit an extension to the full quantum level, i.e. to all orders in $b$, allowing to determine an exact expression for $\kappa(b)$. One important point is the role of the boundary limit: the relation (1.3) between the elementary Liouville field correlators and the stress tensor correlators may hold only at the boundary. A general proof should use the boundary conformal field theory considerations. In particular, the completeness of the OPE in the boundary CFT (cf. appendix D) implies that it contains only the boundary stress tensor and its descendants; since the boundary operator dual to the $m^{2}=2$ Liouville field in $\mathrm{AdS}_{2}$ should have dimension 2 it can only be (proportional to) the stress tensor itself. Considering the OPE of the stress tensor in the bulk and the Liouville field at the

\footnotetext{
${ }^{5}$ As a technical remark, let us note that the relations in (1.9) and in (1.15) are consequences of the main identification (1.3) only. As such they are independent of a particular regularization scheme that one is adopting, provided conformal invariance is respected. In particular, (1.9) and (1.15) will be valid for both schemes that we shall propose in the main text.

${ }^{6}$ This may be compared to the discussion of the conjectured duality between a gravitational theory in $\mathrm{AdS}_{2}$ and a chiral half of a $2 \mathrm{~d} \mathrm{CFT}$ in [20]. While here we assume that the $\mathrm{AdS}_{2}$ background is fixed and the Virasoro symmetry is not gauged, in the gravitational context the $2 \mathrm{~d}$ diffeomorphisms is a gauge symmetry and the Virasoro symmetry (with $c=0$ due to ghosts, etc.) was assumed to be an asymptotic symmetry (also, the Liouville field in [20] was a gravitational mode). Whether this symmetry is spontaneously broken to $\mathrm{SL}(2, \mathbb{R})$ appears to depend on a particular model in question (cf. [21-23]).
} 
boundary should then fix the value of the proportionality constant $\kappa(b)$ or $C_{2}(b)$ in the two-point function in (1.6). ${ }^{7}$

A possible generalization of the above semiclassical argument is as follows. Starting with the Liouville theory on a complex plane, the (normal ordered) operator $V_{a}=e^{2 a \phi}$ is a primary with the conformal dimension $\Delta=a(Q-a)$, i.e. we have

$$
T(w) V_{a}\left(w^{\prime}, \bar{w}^{\prime}\right)=\frac{a(Q-a)}{\left(w-w^{\prime}\right)^{2}} V_{a}\left(w^{\prime}, \bar{w}^{\prime}\right)+\frac{1}{w-w^{\prime}} \partial_{w^{\prime}} V_{a}\left(w^{\prime}, \bar{w}^{\prime}\right)+\cdots .
$$

Expanding this formally in small $a$ gives $T(w) \phi\left(w^{\prime}, \bar{w}^{\prime}\right) \sim \frac{Q}{2\left(w-w^{\prime}\right)^{2}}+\frac{\partial_{w^{\prime}} \phi\left(w^{\prime}, \bar{w}^{\prime}\right)}{w-w^{\prime}}$. On the upper half-plane $(w=t+i \mathbf{z}, \mathbf{z}>0)$ the boundary conformal Ward identity takes into account also the poles at $w=\bar{w}^{\prime}$. Including them in the OPE of $T$ and $\phi$ and assuming the exact form of the boundary asymptotics to be ${ }^{8}$

$$
\left.\phi(t, \mathbf{z})\right|_{z \rightarrow 0}=z^{2} \Phi(t)-Q \log \mathbf{z}+\ldots,
$$

one finds that the leading $\frac{1}{\left(t-t^{\prime}\right)^{2}}$ term in $T(t) \phi(t, 0)$ cancels and we get ${ }^{9}$

$$
T(t) \Phi\left(t^{\prime}\right)=-\frac{2 Q}{\left(t-t^{\prime}\right)^{4}}+\cdots .
$$

Taking the expectation value of (1.19) and comparing the result with (1.4), (1.6), (1.7) we get the following prediction for the exact value of the coefficient $\kappa(b)$ between $\Phi$ and $T$ :

$$
\begin{aligned}
\Phi & =\kappa T, \quad \kappa(b)=-\frac{4 Q}{c}, \\
\kappa(b) & =-\frac{4 Q}{1+6 Q^{2}}=-\frac{4 b\left(1+b^{2}\right)}{\left(3+2 b^{2}\right)\left(2+3 b^{2}\right)}=-\frac{2}{3} b+\frac{7}{9} b^{3}-\frac{55}{54} b^{5}+\cdots .
\end{aligned}
$$

The first two terms in this expansion match precisely with the above semiclassical value and the one-loop value that we shall find below in (3.24). Given (1.20) we get from (1.7)

$$
C_{2}=\frac{8 Q^{2}}{c}=\frac{4}{3}-\frac{2}{9} b^{2}+\ldots, \quad C_{3}=-\frac{64 Q^{3}}{c^{2}}=-\frac{16}{9} b+\frac{64}{27} b^{3}+\ldots,
$$

which are in agreement with (3.25).

A natural generalization of the above duality (1.3) for the Liouville theory is to all conformal Toda theories for finite Lie algebras [24-26] defined on the $\mathrm{AdS}_{2}$ background. For instance, in the $A_{n}$ case, expanding near the minimum of the Toda potential in $\mathrm{AdS}_{2}$ one finds $n$ scalar fields $\varphi_{\Delta}$ with masses $m^{2}=\Delta(\Delta-1)$ corresponding to $\Delta=2, \ldots, n+1[19]$. The duality relation extending (1.3) is then

$$
\left\langle\Phi_{\Delta_{1}}\left(t_{1}\right) \cdots \Phi_{\Delta_{n}}\left(t_{n}\right)\right\rangle=\left.\left(\prod_{i=1}^{n} \kappa_{\Delta_{i}}\right)\left\langle Q_{\Delta_{1}}\left(z_{1}\right) \cdots Q_{\Delta_{n}}\left(z_{n}\right)\right\rangle\right|_{z_{i} \rightarrow t_{i}},
$$

\footnotetext{
${ }^{7}$ We are grateful to Simone Giombi and Xi Yin for useful suggestions on this problem.

${ }^{8}$ This generalizes the classical asymptotics where $Q \rightarrow b^{-1}$ in a way which is consistent with the value of the one-loop tadpole (see [5] and (2.16)).

${ }^{9}$ In more details, eq. (1.19) is the coefficient of $z^{2}$ term in the $z \rightarrow 0$ expansion of $\frac{Q}{2\left(t-t^{\prime}-i z\right)^{2}}+$ $\frac{i}{2} Q \frac{1}{\mathrm{z}} \frac{1}{t-t^{\prime}-i \mathrm{z}}+$ c.c where the second term comes from the $-Q \log \mathrm{z}$ term in (1.18) and the complex conjugate contribution comes from poles at $w=\bar{w}^{\prime}$.
} 
where $Q_{\Delta}=\left\{Q_{2} \equiv T, Q_{3}, \ldots, Q_{n+1}\right\}$ are the generators of the chiral $\mathcal{W}_{n+1}$ algebra generalizing the Virasoro symmetry and having the same central charge as the Toda theory in the bulk. The relation (1.23) was noticed at the tree level in [19] by computing few sample 4-point functions in the Toda theories associated to some rank-2 algebras with two scalar fields (one dual to the stress tensor $T$ and the other dual to a higher spin current $Q_{s}$ ). It is natural to expect (1.23) to hold also at the quantum level as should be possible to check by the methods used in the present paper. Another interesting generalization is to the super-Liouville theory on $\mathrm{AdS}_{2}$, cf. [27]. In this case one will need to compute loop corrections for both the bosonic and fermionic fields.

The structure of the rest of this paper is as follows. In section 2.1 we will discuss two alternative formulations of the Liouville theory on $\mathrm{AdS}_{2}$. One starts with the flat space action expanded around the classical solution corresponding effectively to the $\mathrm{AdS}_{2}$ background while the other starts directly with the Liouville theory defined on fixed $\mathrm{AdS}_{2}$. The two formulations differ in the choice of the regularization of the short distance propagator and also in the coefficient in front of the potential. They turn out to give the equivalent results for the physically relevant connected one-loop correlators.

In section 2.2 we will compute the two-point function of the Liouville field at the one-loop order demonstrating the consistency of its boundary limit with (1.6). The oneloop corrections to the boundary three-point and four-point functions will be computed in sections 3 and 4 respectively finding again the agreement with (1.6) and (1.13). As a result, we will provide a strong support for the validity of the duality (1.3) at the loop level. Appendix A will present some details of the diagram computations on the Poincaré disc. Comments on tadpole diagrams (that lead to different predictions for the 1-point functions in the two formulations) will be made in appendix B. In appendix D we will review the structure of the $1 \mathrm{~d}$ conformal block expansion of the four-point function in (1.5).

\section{Liouville theory on Euclidean $\mathrm{AdS}_{2}$ background}

The correlators in the Liouville theory (1.1) in $\mathrm{AdS}_{2}$ background may be computed using two alternative approaches. In the first (the "ZZ formulation" [5]) one starts with the Liouville action on a flat upper half plane (or flat disc) and expands it near a non-trivial non-constant solution $[1,2]$ preserving the $\operatorname{SL}(2, \mathbb{R})$ symmetry. In the second (the "AdS formulation") one starts directly with the Liouville action (1.1) in the $\mathrm{AdS}_{2}$ background and expands near the constant minimum of the curved-space potential. ${ }^{10}$ The two approaches are classically equivalent by a field redefinition but imply the use of different regularizations at the quantum level.

\footnotetext{
${ }^{10}$ This is similar to the so-called "geometrical" approach [28] discussed in detail in [18].
} 


\subsection{Two alternative formulations}

The flat-space action ${ }^{11}$

$$
S=\frac{1}{4 \pi} \int d^{2} z\left(\partial^{a} \varphi \partial_{a} \varphi+\mu e^{2 b \varphi}\right)
$$

admits a particular solution

$$
\varphi^{(0)}=-\frac{1}{2 b} \log \left[\frac{\mu}{4} b^{2}(1-z \bar{z})^{2}\right] .
$$

Expanding near this "AdS 2 vacuum", $\varphi=\varphi^{(0)}+\chi$, we find the following action for the fluctuation $\chi$

$$
S^{\mathrm{ZZ}}=\frac{1}{2 \pi} \int d^{2} z\left[\frac{1}{2}\left(\partial_{a} \chi\right)^{2}+\frac{2\left(e^{2 b \chi}-2 b \chi-1\right)}{b^{2}(1-z \bar{z})^{2}}\right] .
$$

This action is the same as the classical action (1.1) (with $Q \rightarrow b^{-1}$ ) in $\mathrm{AdS}_{2}$ background in the Poincaré disc (or "pseudosphere") coordinates where $d s^{2}=\frac{4 d z d \bar{z}}{(1-z \bar{z})^{2}}$ that has $\mathrm{SU}(1,1) \simeq$ $\operatorname{SL}(2, \mathbb{R})$ symmetry $\left(z \rightarrow \frac{a z+b}{\bar{b} z+\bar{a}}, \quad|a|^{2}-|b|^{2}=1\right)$. An alternative choice is the half-plane parametrization with coordinates $t \in \mathbb{R}, \mathrm{z} \geq 0$ and the metric $d s^{2}=\frac{1}{\mathrm{z}^{2}}\left(d t^{2}+d \mathrm{z}^{2}\right)$. The two are related by the standard conformal map

$$
w \equiv t+i \mathbf{z}=i \frac{1+z}{1-z}, \quad z=\frac{w-i}{w+i}, \quad d s^{2}=-\frac{4 d w d \bar{w}}{(w-\bar{w})^{2}}=\frac{4 d z d \bar{z}}{(1-z \bar{z})^{2}} .
$$

The analog of the action (2.3) on the Poincaré plane reads

$$
S^{\mathrm{ZZ}}=\frac{1}{2 \pi} \int d t d \mathbf{z}\left[\frac{1}{2}\left(\partial_{a} \chi\right)^{2}+\frac{e^{2 b \chi}-2 b \chi-1}{2 b^{2} \mathbf{z}^{2}}\right] .
$$

As follows from (2.3) or (2.5) the field $\chi$ has the mass $m^{2}=\Delta(\Delta-1)=2$. Assuming the standard Dirichlet boundary condition (corresponding to the $\Delta=2$ choice) the canonically normalized $\mathrm{AdS}_{2}$ bulk-to-bulk propagator is given by

$$
G_{\Delta=2}=\frac{\mathcal{C}_{2}}{16 u^{2}}{ }_{2} F_{1}\left(2,2,4 ;-\frac{1}{u}\right)=-\frac{1}{4 \pi}\left[(1+2 u) \log \frac{u}{u+1}+2\right]
$$

where $\mathcal{C}_{2}=\frac{\Gamma(2)}{2 \sqrt{\pi} \Gamma\left(2+\frac{1}{2}\right)}=\frac{2}{3 \pi}$ and $u$ is the invariant chordal distance. Equivalently (taking into account the factor $\frac{1}{2 \pi}$ in front of the action in (2.3), (2.5)) the free two-point function of the field $\chi$ is given by

$$
\begin{aligned}
g\left(z, z^{\prime}\right) & \equiv\left\langle\chi(z) \chi\left(z^{\prime}\right)\right\rangle_{0}=2 \pi G_{\Delta=2}=-\frac{1}{2}\left(\frac{1+\eta}{1-\eta} \log \eta+2\right), \\
\eta & =\left|\frac{z-z^{\prime}}{1-z \bar{z}^{\prime}}\right|^{2}=\left|\frac{w-w^{\prime}}{w-\bar{w}^{\prime}}\right|^{2}=\frac{u}{u+1}, \quad u=\frac{\left(t-t^{\prime}\right)^{2}+\left(\mathbf{z}-\mathbf{z}^{\prime}\right)^{2}}{4 z z^{\prime}} .
\end{aligned}
$$

\footnotetext{
${ }^{11}$ We shall use the notation $z=x_{1}+i x_{2}, \quad d^{2} z=d x_{1} d x_{2}$. As usual, here one also assumes that the stress tensor of the theory $(2.1)$ is $T=-(\partial \varphi)^{2}+Q \partial^{2} \varphi$ in order to make the interaction term exactly marginal.
} 
Taking one point to the boundary we get the corresponding bulk-to-boundary propagator $g_{\mathrm{b}}$ written in either disc or plane parametrization ${ }^{12}$

$$
\left.\mathrm{z}^{-2} g\left(z, z^{\prime}\right)\right|_{|z| \rightarrow 1} \equiv g_{\mathrm{b}}\left(\theta, z^{\prime}\right)=g_{\mathrm{b}}\left(t, w^{\prime}\right)=\frac{4}{3} \frac{\sin ^{4}\left(\frac{\theta}{2}\right)\left(1-\left|z^{\prime}\right|^{2}\right)^{2}}{\left|e^{i \theta}-z^{\prime}\right|^{4}}=\frac{4}{3}\left[\frac{\mathrm{z}^{\prime}}{\mathrm{z}^{\prime 2}+\left(t-t^{\prime}\right)^{2}}\right]^{2},
$$

where the relation between the Poincaré plane and disc boundary coordinates $t$ and $\theta$ is

$$
t(\theta)=i \frac{1+e^{i \theta}}{1-e^{i \theta}}=-\cot \frac{\theta}{2} .
$$

The starting point of the AdS formulation is the action (1.1) in unit-radius $\mathrm{AdS}_{2}$ background $(R=-2)$. Shifting the field by a constant as $\varphi=\varphi^{(0)}+\chi$ where $\mu e^{2 b \varphi^{(0)}}=\frac{Q}{b}$ we get the following analog of (2.3)

$$
S^{\mathrm{AdS}}=\frac{1}{2 \pi} \int d^{2} z\left[\frac{1}{2}\left(\partial_{a} \chi\right)^{2}+\frac{2 Q\left(e^{2 b \chi}-2 b \chi-1\right)}{b(1-z \bar{z})^{2}}\right] .
$$

The two coincide in the semiclassical limit of small $b$ when $Q=b+b^{-1} \rightarrow b^{-1}$ but differ for finite $b$.

The two formulations will differ also by the required choice of the UV regularization of the propagator (2.7) at the coinciding points but the two differences will happen to compensate each other leading to the equivalent results for the relevant physical correlators (but not for the tadpole values). The ZZ formulation that starts with the flat-space action requires the use of the simple flat-space regularization $z-z^{\prime} \rightarrow z-z^{\prime}+\varepsilon$. Omitting the singular $\log \varepsilon$ term this is equivalent to the subtraction of the $-\left[\log \left(z-z^{\prime}\right)\right]_{z \rightarrow z^{\prime}}$ term from (2.7) [5]. At the same time, the regularization in the AdS formulation should be the $\mathrm{AdS}_{2}$ covariant one with $\eta \rightarrow \eta+\varepsilon$ or $u \rightarrow u+\varepsilon$ in (2.7), (2.8). In general, the value of the propagator at the coinciding points may be parametrized as

$$
\begin{aligned}
g(z, z) & =\mathrm{q}_{1} \log (1-z \bar{z})-\mathrm{q}_{2}, \\
\mathrm{q}_{1}^{\mathrm{ZZ}} & \left.=\mathrm{q}_{2}^{\mathrm{ZZ}}=1, \quad g^{\mathrm{ZZ}}(z, z)=\lim _{z^{\prime} \rightarrow z}\left[g\left(z, z^{\prime}\right)+\log \left|z-z^{\prime}\right|\right)\right]=\log (1-z \bar{z})-1, \\
\mathrm{q}_{1}^{\text {AdS }} & =\mathrm{q}_{2}^{\text {AdS }}=0, \quad g^{\text {AdS }}(z, z)=0 .
\end{aligned}
$$

Here in (2.14) we assumed that the logarithmic UV divergences are cancelled by the mass renormalization with the "minimal" subtraction (more generally, one may keep the constant $\mathrm{q}_{2}^{\text {AdS }}$ arbitrary).

Starting with (2.3) or (2.11) one may compute the corresponding quantum corrections to bulk correlators in perturbation theory in $b$. The two actions differ in the coefficient in front of the potential term

$$
V^{\mathrm{AdS}}=\frac{2 Q}{b}\left(e^{2 b \chi}-2 b \chi-1\right)=\left(1+b^{2}\right) V^{\mathrm{ZZ}},
$$

implying that in the AdS formulation one has extra higher order terms in the $\chi^{2}, \chi^{3}, \ldots$ vertices. In addition, there is a difference in the regularization choices in (2.13), (2.14).

\footnotetext{
${ }^{12}$ The factor $\frac{4}{3}$ is the same as $2 \pi \mathcal{C}_{2}$, where $\mathcal{C}_{2}$ appeared in $(2.6)$.
} 
While the values of the one-point functions $\left\langle\chi^{n}(z)\right\rangle$ computed in the ZZ and AdS formulations will differ, the connected correlators at separated points are expected to be same; we shall find evidence for that below.

\subsection{One-point and two-point correlation functions}

Let us consider the leading order corrections to the tadpole or the one-point function $\langle\chi(z)\rangle$. Computing it for generic choice of the regularization (2.12) one finds (see (A.7),

$$
\langle\chi(z)\rangle=z \bullet \bigcirc+\mathcal{O}\left(b^{3}\right)=b\left[\frac{1}{2} \mathrm{q}_{1}+\mathrm{q}_{2}-\mathrm{q}_{1} \log (1-z \bar{z})\right]+\mathcal{O}\left(b^{3}\right) .
$$

The leading-order tadpole thus vanishes in the AdS formulation and is constant in general, as required by the AdS symmetry (under which $\chi$ transforms as a scalar). Explicitly, one finds

$$
\langle\chi(z)\rangle^{\mathrm{ZZ}}=b\left[\frac{3}{2}-\log (1-z \bar{z})\right]+b^{3}\left(\frac{\pi^{2}}{6}-\frac{13}{12}\right)+\mathcal{O}\left(b^{5}\right), \quad\langle\chi(z)\rangle^{\mathrm{AdS}}=\mathcal{O}\left(b^{3}\right) .
$$

Note that combining the one-loop tadpole with the classical solution (2.2) the $z$-dependent part of the tadpole in the ZZ case gets coefficient $Q=b+b^{-1}$. The $b^{3}$ term in $\langle\chi(z)\rangle^{\mathrm{ZZ}}$ is in agreement with the results of [5] and [29] (see also appendix B). ${ }^{13}$

Next, let us consider the one-loop order $b^{2}$ correction to the connected part of the two-point function $\left\langle\chi(z) \chi\left(z^{\prime}\right)\right\rangle$. In the AdS formulation one needs to take into account an extra contribution coming from the $b^{2} \chi^{2}$ term in the potential in (2.15). The final result in both formulations can be written as

$$
\begin{aligned}
& \left\langle\chi(z) \chi\left(z^{\prime}\right)\right\rangle_{\text {conn }}=\bullet \longrightarrow+\bullet \bigcirc+\mathrm{q}_{1}^{\prime} \bullet \otimes \longrightarrow \mathcal{O}\left(b^{4}\right) \\
& =g\left(z, z^{\prime}\right)+\Sigma\left(z, z^{\prime}\right) \\
& +\frac{2}{3} b^{2} \mathrm{q}_{1}^{\prime}\left[1-\frac{\eta \log \eta}{1-\eta}-\log (1-\eta)\left(1+\frac{(1+\eta) \log \eta}{2(1-\eta)}\right)-\frac{1+\eta}{1-\eta} \operatorname{Li}_{2}(1-\eta)\right]+\mathcal{O}\left(b^{4}\right),
\end{aligned}
$$

where (see (A.11), (A.15), (A.17))

$$
\Sigma\left(z, z^{\prime}\right)=b^{2}\left[\frac{3}{2}+\frac{\eta^{2} \log ^{2} \eta}{2(1-\eta)^{2}}-\frac{1+\eta}{1-\eta} \operatorname{Li}_{2}(1-\eta)\right],
$$

and the coefficient $q_{1}^{\prime}=q_{1}-1$ in the $Z Z$ case and $q_{1}^{\prime}=q_{1}$ in the AdS case. As follows from (2.13), (2.14) in both cases

$$
\mathrm{q}_{1}^{\prime}=0
$$

and so we get the equivalent result for (2.18) given by the sum of the free propagator in (2.7) and the "self-energy" correction in (2.20).

\footnotetext{
${ }^{13}$ The tadpole (2.17) is the simplest cumulant that can be extracted from the expectation value of the exponential vertex operator $\left\langle e^{\alpha \chi(z)}\right\rangle$ determined in [5]. For a perturbative discussion of the $\mathcal{O}\left(b^{3}\right)$ corrections to higher cumulants and a comparison with the bootstrap predictions see [29].
} 
Let us consider the Poincaré plane parametrization and introduce the subtracted and rescaled field

$$
\Phi(t, \mathbf{z})=\mathbf{z}^{-2}[\chi(t, \mathbf{z})-\langle\chi(t, \mathbf{z})\rangle] .
$$

Using (2.7), (2.8), (2.20) and taking the limit $\mathbf{z}_{1}, \mathbf{z}_{2} \rightarrow 0$ we get for the two-point boundary correlator in (1.2) (cf. (2.9))

$$
\left\langle\Phi(t) \Phi\left(t^{\prime}\right)\right\rangle \equiv \lim _{\mathrm{z}, \mathrm{z}^{\prime} \rightarrow 0}\left\langle\chi(t, \mathrm{z}) \chi\left(t^{\prime}, \mathrm{z}^{\prime}\right)\right\rangle_{\mathrm{conn}}=\frac{C_{2}(b)}{\left(t-t^{\prime}\right)^{4}}, \quad C_{2}=\frac{4}{3}\left(1-\frac{1}{6} b^{2}\right)+\mathcal{O}\left(b^{4}\right) .
$$

Thus there are no $b^{2} \log \left(t-t^{\prime}\right)$ corrections, i.e. the boundary operator dual to $\chi$ has no anomalous dimension confirming the expected behaviour (1.6). We conclude that the coefficients in the perturbative expansion of $C_{2}$ introduced in (1.8) are thus

$$
C_{2,0}=\frac{4}{3}, \quad C_{2,1}=-\frac{2}{9} .
$$

Let us note that it is only the boundary limit of the 2-point function (2.18) that takes the simple rational form in (2.23) matching the stress tensor two-point function (1.4) according to (1.3). The same will apply to higher-point correlation functions.

\section{Three-point boundary correlation function}

Next, let us compute the tree-level and one-loop contributions to the connected boundary three-point function. We shall use mainly the $Z Z$ formulation, but will comment on its equivalence with the AdS one. The relevant terms in the expansion of the potential term in (2.3) will be

$$
\int \mathrm{d}^{2} z \frac{e^{2 b \chi}-2 b \chi-1}{b^{2}}=\int \mathrm{d}^{2} z\left[2 \chi^{2}+8 b \frac{\chi^{3}}{3 !}+16 b^{2} \frac{\chi^{4}}{4 !}+32 b^{3} \frac{\chi^{5}}{5 !}+\cdots\right], \quad \mathrm{d}^{2} z \equiv \frac{d^{2} z}{\pi\left(1-|z|^{2}\right)^{2}} .
$$

We will need the tree boundary-to-bulk propagator in the disc parametrization given in (2.9) and also will use the following notation for the conformal prefactor in the threepoint function (1.6) (cf. (2.10))

$$
\mathcal{K}_{3}(\boldsymbol{\theta}) \equiv \mathcal{K}\left(\theta_{1}, \theta_{2}, \theta_{3}\right)=\prod_{i<j}^{3}\left|t\left(\theta_{i}\right)-t\left(\theta_{j}\right)\right|^{-2} .
$$

We shall divide the relevant Witten diagrams in $\mathrm{AdS}_{2}$ by the overall conformal factor $\mathcal{K}_{3}$, i.e. the resulting expressions will represent the contributions to the coefficients in $C_{3}(b)$ in (1.6).

The tree level contribution to the three-point function is given by the diagram

$$
C_{3,0} b=\overbrace{-\ldots . . .}^{-}
$$


The same result in the Poincaré plane coordinates follows from (see, e.g., [30] ${ }^{14}$

$$
\int \frac{d t d z}{z^{2}} \prod_{i=1}^{3}\left[\frac{z}{z^{2}+\left(t-t_{i}\right)^{2}}\right]^{2}=\frac{3 \pi}{8} \frac{1}{\left|t_{12}\right|^{2}\left|t_{13}\right|^{2}\left|t_{23}\right|^{2}} .
$$

Thus the value of the leading coefficient in $C_{3}$ in (1.6), (1.8) is

$$
C_{3,0}=-\frac{16}{9}
$$

which together with $C_{2,0}$ in (2.24) is in agreement with the first relation in (1.9).

The value (3.5) combined with (2.24) and the second relation in (1.9) gives the following prediction for the one-loop coefficient $C_{3,1}$ in $C_{3}(b)$ in (1.8)

$$
C_{3,1}=\frac{64}{27}
$$

This coefficient should be given by the sum of the contributions of the four different types of diagrams

$$
C_{3,1}=\sum_{i=1}^{4} C_{3,1}^{(i)}
$$

that we shall consider below.

Diagrams with dressed propagators. There are three tree-like diagrams with the one-loop self-energy correction to one of the propagators

$$
C_{3,1}^{(1)} b^{3}=\overbrace{\cdots, \ldots}^{i}
$$

The one-loop corrected propagator is given by (2.18), (2.20) (and is the same in both ZZ and AdS formulations). As a result, like in (2.23), the contribution of each of the diagrams in (3.8) is given by the tree-level expression (3.3) times the $1-\frac{1}{6} b^{2}$ factor, i.e.

$$
C_{3,1}^{(1)}=C_{3,0} \times 3 \times\left(-\frac{1}{6}\right)=\frac{8}{9} .
$$

Diagrams with vertex tadpoles. In the ZZ formulation the contribution of diagrams with tadpoles attached to an internal 3 -vertex

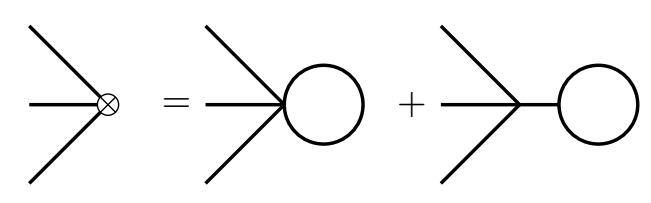

\footnotetext{
${ }^{14}$ See $(2.9)$ and note that $\frac{d t d z}{z^{2}}=\frac{4 d^{2} z}{\left(1-|z|^{2}\right)^{2}}$.
} 
turns out to be equivalent to that of the tree diagram (3.3) with the cubic vertex $8 b^{3} \frac{\chi^{3}}{3 !}$ (the same as in $(3.1)$ with $\left.b \rightarrow b^{3}\right)^{15}$

$$
C_{3,1}^{(2)} b^{3}=\overbrace{-. . .-}^{\prime}
$$

In the AdS formulation we get the same result (by a mechanism similar to the one in the case of the two-point function (2.18)-(2.21)). Here the tadpole contributions vanish $\left(g(z, z)=0\right.$ in (2.14)) but the cubic vertex $\sim b \chi^{3}$ in (3.1) is rescaled by $b Q=1+b^{2}$ (see $(2.15)$ ), i.e. $-8 b \chi^{3} \rightarrow-8 b \chi^{3}-8 b^{3} \chi^{3}$ resulting in an additional one-loop correction. Thus

$$
C_{3,1}^{(2)}=C_{3,0}=-\frac{16}{9}
$$

Diagrams with cubic and quartic vertices. There are also one-loop diagrams with one cubic and one quartic vertices from (3.1)

$$
C_{3,1}^{(3)} b^{3}=\overbrace{-. .}^{\prime}
$$

Explicitly, we get (cf. (3.3))

$$
\overbrace{\theta_{2}}^{\theta_{1}} \overbrace{i}^{\prime}=\frac{\frac{1}{2}(-8 b)\left(-16 b^{2}\right)}{\mathcal{K}_{3}(\boldsymbol{\theta})} \int \mathrm{d}^{2} z^{\prime} \mathrm{d}^{2} z^{\prime \prime} g_{\mathrm{b}}\left(\theta_{1}, z^{\prime}\right) g_{\mathrm{b}}\left(\theta_{2}, z^{\prime \prime}\right) g_{\mathrm{b}}\left(\theta_{3}, z^{\prime \prime}\right)\left[g\left(z^{\prime}, z^{\prime \prime}\right)\right]^{2},
$$

where $\frac{1}{2}$ is the leg symmetry factor. Part of this diagram (the loop with upper leg and integration over the cubic vertex point) is the same as the function $\mathrm{B}\left(z_{2}, z_{1}\right)=$ $\int \mathrm{d}^{2} z^{\prime} g\left(z^{\prime}, z_{1}\right)\left[g\left(z_{2}, z^{\prime}\right)\right]^{2}$ in (A.11), (A.14). Taking the point $z_{1}$ to the boundary then gives, according to (A.14), the boundary-to-bulk propagator (2.9) times the $\frac{1}{8}$ factor

$$
\lim _{\left|z_{1}\right| \rightarrow 1} \frac{1}{z_{1}^{2}} \quad z_{1} \bullet z_{2}=\frac{1}{8} \theta_{1} \longmapsto z_{2}
$$

This should be multiplied by the coupling constants from the two vertices $(-8 b)\left(-16 b^{2}\right)$, by a symmetry factor $\frac{1}{2}$ and by the diagram multiplicity factor 3 . Compared to the tree diagram contribution this gives an extra $-3 b^{2}$ factor in total, i.e.

$$
C_{3,1}^{(3)}=-3 C_{3,0}=\frac{16}{3} .
$$

\footnotetext{
${ }^{15}$ In the $\mathrm{ZZ}$ formulation $g(z, z)$ is given by (2.13) and taking into account the combinatorics (see (A.9), (A.10)) the contribution is found to be the same as of the tree diagram but with the coupling factor $-8 b$ in (3.3) replaced by $-8 b^{3}$. The same expression appears in eq. (15) of [29].
} 


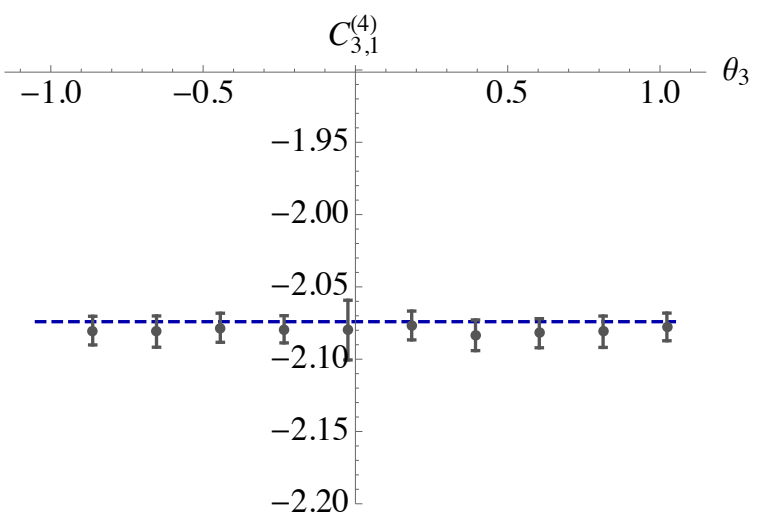

Figure 1. Numerical estimate of $C_{3,1}^{(4)}$ extracted by $10^{7}$ integrand evaluations for $\theta_{1}=\frac{2 \pi}{3}, \theta_{2}=-\frac{2 \pi}{3}$ and variable $\theta_{3}$ with $\left|\theta_{3}\right|<\frac{\pi}{3}$. The horizontal blue line is the expected value $-\frac{56}{27}$.

Diagram with one-loop vertex correction. The most complicated diagram is the one with the three $\chi^{3}$ vertices and thus with the three bulk-to-bulk and the three bulk-toboundary propagators:

$$
C_{3,1}^{(4)} b^{3}=\overbrace{-\ldots . . ;}^{\prime}=\frac{(-8 b)^{3}}{\mathcal{K}_{3}(\boldsymbol{\theta})} \int \mathrm{d}^{2} z_{1} \mathrm{~d}^{2} z_{2} \mathrm{~d}^{2} z_{3} g\left(z_{1}, z_{2}\right) g\left(z_{1}, z_{3}\right) g\left(z_{2}, z_{3}\right) \prod_{i=1}^{3} g_{\mathrm{b}}\left(\theta_{i}, z_{i}\right) .
$$

As this is the triple $\mathrm{AdS}_{2}$ integral its analytic computation is non-trivial. According to (3.6), (3.7) and the results in (3.9), (3.12), (3.16) the expected value of $C_{3,1}^{(4)}$ should be

$$
C_{3,1}^{(4)}=\frac{64}{27}-\frac{8}{9}+\frac{16}{9}-\frac{16}{3}=-\frac{56}{27} .
$$

Since (3.17) should give just the value of the constant $C_{3,1}^{(4)}$ it is sufficient to evaluate it numerically. We did this by using the Suave routine of the Cuba library [31]. One important point is to check that $C_{3,1}^{(4)}$ given by (3.17) is indeed independent of the boundary coordinates $\theta_{1}, \theta_{2}, \theta_{3}$. From a practical perspective, one is interested in the $\theta_{i}$ dependence of the systematic error in the numerical estimate at fixed computational effort (the total number of integrand evaluations). In figure 1 we fixed the two points at $\theta_{1}=\frac{2 \pi}{3}, \theta_{2}=-\frac{2 \pi}{3}$ and varied $\theta_{3}$ showing the resulting numerical estimate for $C_{3,1}^{(4)}$. The horizontal line is the expected value $-\frac{56}{27}$ in (3.18). One finds that the integral value is more "flat" when the three external points $\theta_{i}$ are well separated. This is expected as the integrand has a more singular behaviour when at least two external points get close. Considering the equally spaced points and increasing statistics we found the best estimate to be ${ }^{16}$

$$
C_{3,1}^{(4)}=-2.08(2) \text {. }
$$

This is in good agreement with $(3.18)$ or $-\frac{56}{27} \approx-2.0741$.

\footnotetext{
${ }^{16}$ The number in the parenthesis is the statistical uncertainty (the least significant digits of a given numerical value for the average). Here the uncertainty is the standard deviation of the Monte-Carlo evaluation of the loop integral. Explicitly, 2.08(2) $\equiv 2.08 \pm 0.02$.
} 
The expected value (3.6) and thus (3.18) was found from (1.9) which was based on matching the two-point and three-point correlators (1.2) with the stress tensor ones (1.4) for the Liouville central charge value $c=1+6\left(b+b^{-1}\right)^{2}$. Alternatively, we may determine the values of $c$ and $\kappa$ in (1.7) from the explicit tree plus one-loop results for $C_{2}$ and $C_{3}$ found above. Omitting the overall conformally covariant factors we may symbolically summarize the results for the two-point and three-point correlators in (2.23) and (3.5), (3.9), (3.10), (3.16), (3.19) as follows

$$
\langle\Phi \Phi\rangle=\frac{4}{3}\left(1-\frac{1}{6} b^{2}\right)+\mathcal{O}\left(b^{4}\right), \quad\langle\Phi \Phi \Phi\rangle=-\frac{16}{9} b+\left(\frac{8}{9}-\frac{16}{9}+\frac{16}{3}+C_{3,1}^{(4)}\right) b^{3}+\mathcal{O}\left(b^{5}\right) .
$$

The consistency of (3.20) with the identification in (1.3), (1.4) or (1.7)

$$
\langle\Phi \Phi\rangle=\frac{1}{2} c \kappa^{2}, \quad\langle\Phi \Phi \Phi\rangle=c \kappa^{3}
$$

implies that

$$
\kappa=-\frac{2}{3} b+\left(\frac{14}{9}+\frac{3}{8} C_{3,1}^{(4)}\right) b^{3}+\mathcal{O}\left(b^{5}\right), \quad c=6 b^{-2}+27\left(1+\frac{1}{4} C_{3,1}^{(4)}\right)+\mathcal{O}\left(b^{2}\right) .
$$

Using the numerical estimate for $C_{3,1}^{(4)}$ found in (3.19) gives

$$
c=6 b^{-2}+13.0(1)+\mathcal{O}\left(b^{2}\right),
$$

which is in good agreement with the value of the Liouville central charge $c=6 b^{-2}+13+6 b^{2}$. Note also that for $C_{3,1}^{(4)}=-\frac{56}{27}$ corresponding to the exact value of $c$ we get

$$
\kappa=-\frac{2}{3} b\left(1-\frac{7}{6} b^{2}\right)+\mathcal{O}\left(b^{5}\right),
$$

and thus the following expressions for $C_{2}(2.23)$ and $C_{3}$ in (1.6), (1.8)

$$
C_{2}=\frac{4}{3}\left(1-\frac{1}{6} b^{2}\right)+\mathcal{O}\left(b^{4}\right), \quad C_{3}=-\frac{16}{9} b\left(1-\frac{4}{3} b^{2}\right)+\mathcal{O}\left(b^{5}\right) .
$$

\section{Four-point boundary correlation function}

The four-point function (1.11) receives contributions from the disconnected and connected diagrams in $\mathrm{AdS}_{2}$. The disconnected part is given by $\left\langle\Phi\left(t_{1}\right) \Phi\left(t_{2}\right)\right\rangle\left\langle\Phi\left(t_{3}\right) \Phi\left(t_{4}\right)\right\rangle$ ( + permutations) corresponding to (1.12). From (3.21), (4.1) the prediction for the non-trivial connected part is

$$
\begin{aligned}
\left\langle\Phi\left(t_{1}\right) \Phi\left(t_{2}\right) \Phi\left(t_{3}\right) \Phi\left(t_{4}\right)\right\rangle_{\mathrm{conn}} & =C_{4}(b)\left(\frac{1}{t_{12}^{2} t_{23}^{2} t_{34}^{2} t_{14}^{2}}+\frac{1}{t_{13}^{2} t_{24}^{2} t_{14}^{2} t_{23}^{2}}+\frac{1}{t_{12}^{2} t_{24}^{2} t_{34}^{2} t_{13}^{2}}\right), \\
C_{4}(b) & =c \kappa^{4}=\frac{32}{27} b^{2}-\frac{80}{27} b^{4}+\mathcal{O}\left(b^{6}\right),
\end{aligned}
$$

implying that in (1.14) one should have

$$
C_{4,0}=\frac{32}{27}, \quad C_{4,1}=-\frac{80}{27} .
$$


These values are in agreement with the relations in (1.15) for the coefficients $C_{2,0}=\frac{4}{3}$, $C_{2,1}=-\frac{2}{9}, C_{3,0}=-\frac{16}{9}, C_{3,1}=\frac{64}{27}$ found above.

The value of the leading coefficient $C_{4,0}$ follows from the sum of the tree-level contact diagram and the three exchange diagrams as in (1.10) (see appendix $\mathrm{C}$ )

$$
C_{4,0} b^{2}=\left(C_{4,0}^{\text {cont }}+C_{4,0}^{\text {exch }}\right) b^{2}=
$$

The one-loop coefficient $C_{4,1}$ is determined from the sum of the contributions of the five classes of diagrams

$$
C_{4,1}=C_{4,1}^{(0)}+\sum_{i=1}^{4} C_{4,1}^{(i)}
$$

To compute them we shall use the AdS formulation in which there are no tadpoles but the potential has an extra factor $\left(1+b^{2}\right)$ in $(2.15)$ rescaling the vertices. $C_{4,1}^{(0)}$ will denote the resulting contribution originating, due to this rescaling, from the contact and exchange tree diagrams in (4.4). Since the contact 4 -vertex is rescaled by $1+b^{2}$ while the exchange diagrams with two 3 -vertices by $\left(1+b^{2}\right)^{2}$ the two are combined now with the relative factor of 2 (cf. (4.4))

$$
C_{4,1}^{(0)}=C_{4,0}^{\text {cont }}+2 C_{4,0}^{\text {exch }}
$$

The contributions $C_{4,1}^{(i)}$ with $i=1,2,3,4$ in (4.5) come from the genuine one-loop diagrams described below.

In writing (4.5) and (4.6) we formally assumed that $t_{i}$-dependence of each individual diagram contribution is given by the same simple conformal factor as in (4.1) (cf. (3.2), (2.10))

$$
\mathcal{K}_{4}(\boldsymbol{\theta}) \equiv \mathcal{K}_{4}\left(\theta_{1}, \ldots, \theta_{4}\right)=\frac{1}{t_{12}^{2} t_{23}^{2} t_{34}^{2} t_{14}^{2}}+\frac{1}{t_{13}^{2} t_{24}^{2} t_{14}^{2} t_{23}^{2}}+\frac{1}{t_{12}^{2} t_{24}^{2} t_{34}^{2} t_{13}^{2}}, \quad t_{i j}=t\left(\theta_{i}\right)-t\left(\theta_{j}\right),
$$

However, this need not be true in general. Let us first recall that the tree level fourpoint boundary correlator (1.10) has the canonical form in (1.12), (1.13) without any logarithmic terms depending on the $1 \mathrm{~d}$ invariant cross-ratio $\chi=\frac{t_{12} t_{34}}{t_{13} t_{24}}$. Such terms cancel between the contact and exchange diagram contributions [19] (see appendix C) but they survive if the contact and exchange contributions are combined with a different relative coefficient like in (4.6) (see (C.2)). These extra terms should still not appear in the total expression (4.1) for the one-loop corrected correlator, i.e. they should cancel against similar "extra" contributions of other diagrams. Checking this directly would require the analytic computation of all the diagrams discussed below which we will not attempt here.

Here we will compute the total value of the coefficient $C_{4,1}$ numerically by fixing particular values of the boundary coordinates $t_{i}$. We will also give the values of the individual diagram contributions $C_{4,1}^{(k)}$ defined formally as the corresponding integrals divided by the factor $\mathcal{K}_{4}$ in (4.7). 
Diagrams with dressed external propagators. These diagrams are analogous to the ones in (3.8) (their contribution is actually proportional to $K_{4}(\boldsymbol{\theta})$ ). As in (3.8), (3.9) the one-loop dressing of the bulk-to-boundary propagators gives the contribution

$$
C_{4,1}^{(1)}=C_{4,0} \times 4 \times\left(-\frac{1}{6}\right)=-\frac{64}{81} .
$$

Diagrams with dressed internal propagator.

$$
C_{4,1}^{(2)} b^{4}=\overbrace{-. . .}
$$

Here the grey circle stands for the one-loop self energy correction $\Sigma\left(z, z^{\prime}\right)$ in $(2.20)$ or explicitly

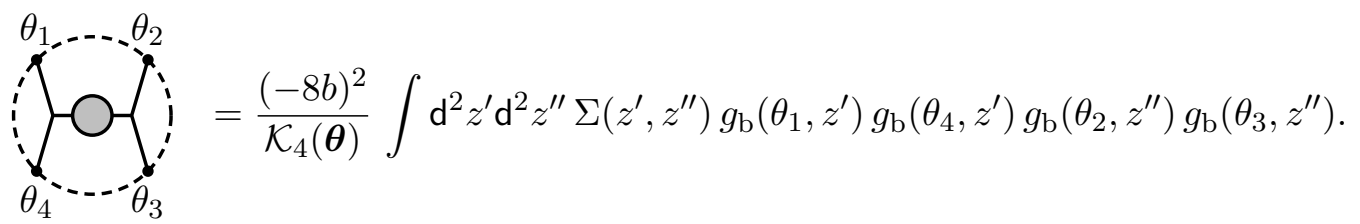

Diagrams with one-loop 1PI cubic vertex correction.

$$
C_{4,1}^{(3)} b^{4}=
$$

Here the grey circle stands for the one-loop cubic vertex correction. In the AdS formulation where the tadpoles are absent it is given by the 1PI diagrams

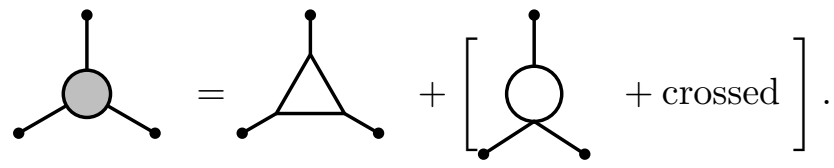

The expressions for the resulting integrals are similar to the one-loop three-point contributions in (3.14) and (3.17) connected to the remaining tree parts.

Diagrams with 1PI quartic vertex corrections. The final class of diagrams represent irreducible quartic vertex corrections (here we do not indicate explicitly additional diagrams obtained by exchanging the boundary points)

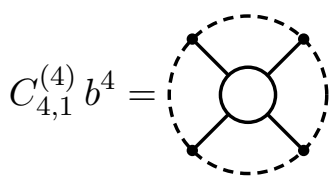

(a)

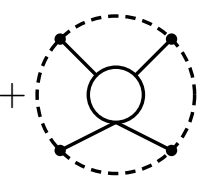

(b)

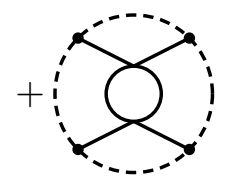

(c)

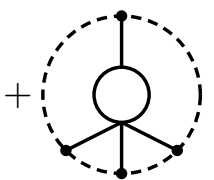

(d) 
The integrals corresponding to these diagrams are

$$
=\frac{(-8 b)^{4}}{\mathcal{K}_{4}(\boldsymbol{\theta})} \int \prod_{i=1}^{4} \mathrm{~d}^{2} z_{i} \prod_{i=1}^{4} g_{\mathrm{b}}\left(\theta_{i}, z_{i}\right) g\left(z_{1}, z_{2}\right) g\left(z_{2}, z_{3}\right) g\left(z_{3}, z_{4}\right) g\left(z_{1}, z_{4}\right),
$$

We evaluated $C_{4,1}^{(i)}$ by the numerical integration for the boundary point configurations where the four $\theta_{i}$ are almost equally spaced. As was discussed above in the case of the threepoint function, such choice is expected to minimize the systematic numerical error. The integrator routine is again chosen to be Suave. The most challenging is the computation of $C_{4,1}^{(3)}$ in (4.11) for which we evaluated the integrand at about $10^{8}$ points. To give an idea of the relative weight of the different contributions, we quote them for the symmetric configuration $\boldsymbol{\theta}=\left(\frac{\pi}{4}, \frac{3 \pi}{4}, \frac{5 \pi}{4}, \frac{7 \pi}{4}\right)$

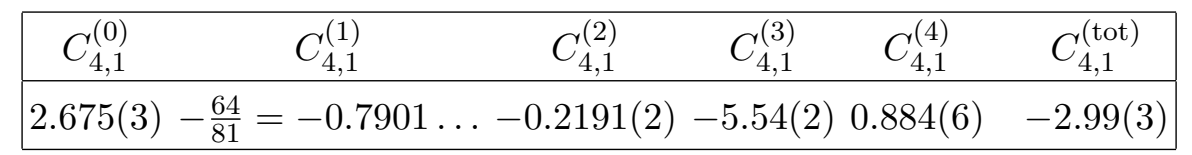

The resulting total value $C_{4,1}^{(\text {tot })}=-2.99 \pm 0.03$ given by the sum in (4.5) is to be compared with the expected value in $(4.2)$, i.e. $C_{4,1}=-\frac{80}{27}=-2.963 \ldots$ The relative error is thus below the $1 \%$ level and within the statistical uncertainty of the multidimensional integration. This very good agreement with the prediction (4.3) effectively confirms our assumption that like the tree-level one, the total one-loop correlator has simple dependence on $t_{i}$ as in (4.1). 


\section{Acknowledgments}

We are grateful to Simone Giombi, Erik Tonni and Xi Yin for very useful discussions. AAT was supported by the STFC grant ST/P000762/1. AAT also thanks the Galileo Galilei Institute for Theoretical Physics for hospitality and INFN for partial support during the completion of this work.

\section{A $\quad \mathrm{AdS}_{2}$ integrals in disc parametrization}

Below we collect some results about the $\mathrm{SU}(1,1)$ covariant integrals over the unit disc that were used in the main text. We shall use the invariant measure

$$
\mathrm{d}^{2} z=\frac{d^{2} z}{\pi\left(1-|z|^{2}\right)^{2}},
$$

and the propagator $g\left(z, z^{\prime}\right)$ defined in (2.7) with $g(z, z)$ parametrized as in (2.12). A very useful representation of the propagator is the Fourier expansion derived in [29]. Denoting by $\vartheta\left(z, z^{\prime}\right)$ the angle between the disc points $z, z^{\prime}$, one has

$$
\begin{array}{rlrl}
g\left(z, z^{\prime}\right) & =\sum_{n=0}^{\infty} g_{n}\left(|z|^{2},\left|z^{\prime}\right|^{2}\right) \cos \left(n \vartheta\left(z, z^{\prime}\right)\right), \\
g_{n}(x, y) & =\theta(y-x) a_{n}(x) b_{n}(y)+\theta(x-y) a_{n}(y) b_{n}(x), \\
a_{0}(x) & =\frac{1+x}{1-x}, \\
a_{1}(x) & =\frac{\sqrt{x}}{1-x}, \\
b_{0}(y) & =-\frac{1}{2}\left(\frac{1+y}{1-y} \log y+2\right), & b_{1}(y) & =\frac{1}{\sqrt{y}}\left(\frac{2 y}{1-y} \log y+1+y\right), \\
a_{n \geq 2}(x) & =\frac{x^{\frac{n}{2}}}{1-x}\left(1-\frac{n-1}{n+1} x\right), & b_{n \geq 2}(y) & =-\frac{y^{-\frac{n}{2}}}{n(n-1)}\left(\frac{1+y}{1-y}\left(1-y^{n}\right)-n\left(1+y^{n}\right)\right),
\end{array}
$$

where $\theta(y-x)$ in (A.3) is the step function.

\section{A.1 Tadpole reduction identity}

Let us consider the integral (using (2.12))

$$
I(z)=\int \mathrm{d}^{2} z^{\prime} g\left(z, z^{\prime}\right) g\left(z^{\prime}, z^{\prime}\right)=\int \mathrm{d}^{2} z^{\prime} g\left(z, z^{\prime}\right)\left[\mathrm{q}_{1} \log \left(1-\left|z^{\prime}\right|^{2}\right)-\mathrm{q}_{2}\right] .
$$

Substituting (A.2) the only contributing term is $n=0$. After integrating over the angle and setting $\left|z^{\prime}\right|=r$ we have

$$
\begin{aligned}
I(z)= & 2 \pi \int_{0}^{1} \frac{r d r}{\pi\left(1-r^{2}\right)^{2}} g_{0}\left(|z|^{2}, r^{2}\right)\left[\mathrm{q}_{1} \log \left(1-r^{2}\right)-\mathrm{q}_{2}\right] \\
= & 2 \frac{1+|z|^{2}}{1-|z|^{2}} \int_{|z|}^{1} \frac{r d r}{\left(1-r^{2}\right)^{2}} b_{0}\left(r^{2}\right)\left[\mathrm{q}_{1} \log \left(1-r^{2}\right)-\mathrm{q}_{2}\right] \\
& +2 b_{0}\left(|z|^{2}\right) \int_{0}^{|z|} \frac{r d r}{\left(1-r^{2}\right)^{2}} \frac{1+r^{2}}{1-r^{2}}\left[\mathrm{q}_{1} \log \left(1-r^{2}\right)-\mathrm{q}_{2}\right] .
\end{aligned}
$$


Integrating over $x=r^{2}$ gives

$$
I(z)=-\frac{1}{8}\left(\mathrm{q}_{1}+2 \mathrm{q}_{2}\right)+\frac{1}{4} \mathrm{q}_{1} \log \left(1-|z|^{2}\right) .
$$

Combining (A.5) and (A.7) we get a simple relation (which follows also from the defining equation for the propagator)

$$
g(z, z)-4 \int \mathrm{d}^{2} z^{\prime} g\left(z, z^{\prime}\right) g\left(z^{\prime}, z^{\prime}\right)=\frac{\mathrm{q}_{1}}{2} .
$$

It may be used to perform the following reduction of the tadpole diagrams (special cases of which are mentioned in [29])

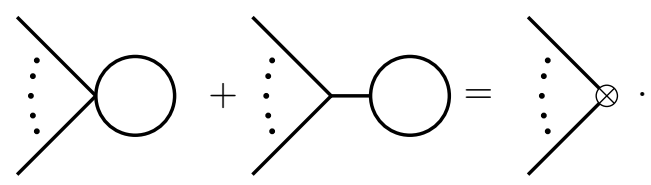

Here dots stand for $N$ external lines and the crossed vertex in the r.h.s. corresponds to the interaction vertex $\sim \chi^{N}$. Its precise coefficient may be determined as follows. The explicit form of the l.h.s. of (A.9) is

$$
-\frac{1}{2} \frac{1}{b^{2}}(2 b)^{N+2} g(z, z)+\frac{1}{2} \frac{1}{b^{2}}(2 b)^{N+1} \frac{1}{b^{2}}(2 b)^{3} \int \mathrm{d}^{2} z^{\prime} g\left(z, z^{\prime}\right) g\left(z^{\prime}, z^{\prime}\right)=-\mathrm{q}_{1}(2 b)^{N},
$$

where we used (A.8) and took into account the $\frac{1}{2}$ symmetry factor due to the tadpoles. In the ZZ formulation we have $\mathrm{q}_{1}^{\mathrm{ZZ}}=1$ (see (2.13)) and thus the r.h.s. corresponds to $(2 b)^{N} \frac{\chi^{N}}{N !}$, i.e. $b^{2}$ times the interaction term in the ZZ Lagrangian (2.3). Thus the one-loop tadpoles in the $\mathbf{Z Z}$ formulation give the same contribution as the extra couplings in (2.15) in the AdS formulation.

\section{A.2 One-loop correction to the two-point function}

The one-loop correction to the connected two-point function is the sum of the bubble diagram

$$
\begin{aligned}
D\left(z_{1}, z_{2}\right) & =z_{1} \longrightarrow z_{2}=\int \mathrm{d}^{2} z^{\prime} \mathrm{d}^{2} z^{\prime \prime} g\left(z_{1}, z^{\prime}\right)\left[g\left(z^{\prime}, z^{\prime \prime}\right)\right]^{2} g\left(z^{\prime \prime}, z_{2}\right) \\
& =\int \mathrm{d}^{2} z^{\prime} g\left(z_{1}, z^{\prime}\right) \mathrm{B}\left(z^{\prime}, z_{2}\right), \quad \quad \mathrm{B}\left(z_{1}, z_{2}\right)=\int \mathrm{d}^{2} z^{\prime}\left[g\left(z_{1}, z^{\prime}\right)\right]^{2} g\left(z^{\prime}, z_{2}\right),
\end{aligned}
$$

plus the tadpole diagrams contribution (A.9) that, using (A.8), (A.10), is given by $\mathrm{q}_{1} \widehat{\mathrm{B}}\left(z_{1}, z_{2}\right)$ with

$$
\widehat{\mathrm{B}}\left(z_{1}, z_{2}\right)=\int \mathrm{d}^{2} z^{\prime} g\left(z_{1}, z^{\prime}\right) g\left(z^{\prime}, z_{2}\right) .
$$

Let us begin with the evaluation of $B\left(z_{1}, z_{2}\right)$. The result should be the function of the $\mathrm{SU}(1,1)$ invariant $\eta\left(z, z^{\prime}\right)=\left|\frac{z-z^{\prime}}{1-z \bar{z}^{\prime}}\right|^{2}$ in $(2.8)$. We may use the 3 parameters of $\mathrm{SU}(1,1)$ to 
first set $z_{1}=0$ and $z_{2}=R \in \mathbb{R}$ and then identify $R^{2}$ with $\eta$ in the final result. ${ }^{17}$ Denoting $g(z, 0) \equiv g\left(|z|^{2}\right)$ and $x=\left|z^{\prime}\right|^{2}$, we get (see (A.3) for the expression for $g_{0}$ )

$$
\begin{aligned}
\mathrm{B}(0, R) & =\int \mathrm{d}^{2} z^{\prime}\left[g\left(\left|z^{\prime}\right|^{2}\right)\right]^{2} g\left(z^{\prime}, R\right)=\int_{0}^{1} \frac{d x}{(1-x)^{2}}[g(x)]^{2} g_{0}\left(x, R^{2}\right) \\
& =b_{0}\left(R^{2}\right) \int_{0}^{R^{2}} \frac{d x}{(1-x)^{2}}[g(x)]^{2} a_{0}(x)+a_{0}\left(R^{2}\right) \int_{R^{2}}^{1} \frac{d x}{(1-x)^{2}} g(x)^{2} b_{0}(x) \\
& =\frac{1}{8}-\frac{R^{2} \log ^{2}\left(R^{2}\right)}{8\left(R^{2}-1\right)^{2}}
\end{aligned}
$$

The result is thus [5]

$$
\mathrm{B}\left(z_{1}, z_{2}\right)=\frac{1}{8}-\frac{\eta \log ^{2} \eta}{8(1-\eta)^{2}}, \quad \eta=\eta\left(z_{1}, z_{2}\right)
$$

It is then straightforward to find the expression for $D\left(z_{1}, z_{2}\right)$ in (A.11). We choose again $z_{1}=R>0$ and $z_{2}=0$ to get

$$
\begin{aligned}
D\left(z_{1}, z_{2}\right)= & D(R, 0)=\int \mathrm{d}^{2} z^{\prime} g\left(R, z^{\prime}\right) \mathrm{B}\left(z^{\prime}, 0\right)=\int \mathrm{d}^{2} z^{\prime} g\left(R, z^{\prime}\right)\left[\frac{1}{8}-\frac{\left|z^{\prime}\right|^{2} \log ^{2}\left(\left|z^{\prime}\right|^{2}\right)}{8\left(1-\left|z^{\prime}\right|^{2}\right)^{2}}\right] \\
= & \int_{0}^{1} \frac{d x}{(1-x)^{2}} g_{0}\left(R^{2}, x\right)\left[\frac{1}{8}-\frac{x \log ^{2} x}{8(1-x)^{2}}\right] \\
= & b_{0}\left(R^{2}\right) \int_{0}^{R^{2}} \frac{d x}{(1-x)^{2}} a_{0}(x)\left[\frac{1}{8}-\frac{x \log ^{2} x}{8(1-x)^{2}}\right] \\
& +a_{0}\left(R^{2}\right) \int_{R^{2}}^{1} \frac{d x}{(1-x)^{2}} b_{0}(x)\left[\frac{1}{8}-\frac{x \log ^{2} x}{8(1-x)^{2}}\right] \\
= & \frac{\eta^{2} \log ^{2} \eta}{64(\eta-1)^{2}}-\frac{4 \eta+9}{96(\eta-1)}-\frac{\eta \log \eta}{48(\eta-1)}+\log (1-\eta)\left[\frac{1}{48}-\frac{(\eta+1) \log \eta}{48(\eta-1)}\right] \\
& -\frac{(\eta+1) \operatorname{Li}_{2}(\eta)}{96(\eta-1)}-\frac{39+\pi^{2}}{576} \frac{1+\eta}{1-\eta},
\end{aligned}
$$

where in the last step we replaced $R^{2}$ by $\eta$. The tadpole contribution (A.12) is worked out as in (A.13), (A.15). We get

$$
\begin{aligned}
\widehat{\mathrm{B}}(R, 0) & =\int \mathrm{d}^{2} z^{\prime} g\left(R, z^{\prime}\right) g\left(z^{\prime}, 0\right)=\int \mathrm{d}^{2} z^{\prime} g\left(R, z^{\prime}\right) g\left(\left|z^{\prime}\right|^{2}\right)=\int_{0}^{1} \frac{d x}{(1-x)^{2}} g_{0}\left(R^{2}, x\right) g(x) \\
& =b_{0}\left(R^{2}\right) \int_{0}^{R^{2}} \frac{d x}{(1-x)^{2}} a_{0}(x) g(x)+a_{0}\left(R^{2}\right) \int_{R^{2}}^{1} \frac{d x}{(1-x)^{2}} b_{0}(x) g(x) \\
& =-\frac{\eta \log \eta}{6(\eta-1)}+\log (1-\eta)\left[\frac{1}{6}-\frac{(\eta+1) \log \eta}{12(\eta-1)}\right]-\frac{(\eta+1) \operatorname{Li}_{2}(1-\eta)}{6(\eta-1)}-\frac{1}{6} .
\end{aligned}
$$

\footnotetext{
${ }^{17}$ One can always find an $\mathrm{SU}(1,1)$ transformation sending $z_{2} \rightarrow R>0$ and $z_{1} \rightarrow 0$ : the corresponding parameters are $b=-a z_{1},|a|=1 /\left(1-\left|z_{1}\right|^{2}\right)$. From $R=\left(a z_{2}-a z_{1}\right) /\left(-\bar{a} \bar{z}_{1} z_{2}+\bar{a}\right)=\frac{a}{\bar{a}}\left(z_{2}-z_{1}\right) /\left(1-z_{2} \bar{z}_{1}\right)$ we find $R^{2}=\eta\left(z_{2}, z_{1}\right)$ and determine also $\arg a$.
} 
Taking into account the combinatoric coefficients and the couplings ${ }^{18}$ the total one-loop correction to the connected part of the two-point function is (cf. (2.18))

$$
\begin{aligned}
& 32 D\left(z_{1}, z_{2}\right)-4 \mathrm{q}_{1} \widehat{\mathrm{B}}\left(z_{1}, z_{2}\right)=\frac{3}{2}+\frac{\eta^{2} \log ^{2} \eta}{2(1-\eta)^{2}}-\frac{1+\eta}{1-\eta} \mathrm{Li}_{2}(1-\eta) \\
& +\frac{2}{3}\left(\mathrm{q}_{1}-1\right)\left(1-\frac{\eta \log \eta}{1-\eta}-\log (1-\eta)\left[1+\frac{(1+\eta) \log \eta}{2(1-\eta)}\right]-\frac{1+\eta}{1-\eta} \operatorname{Li}_{2}(1-\eta)\right) .
\end{aligned}
$$

For $\mathrm{q}_{1}=1$ this gives $\Sigma\left(z_{1}, z_{2}\right)$ in $(2.20)$ and agrees with the result of [18]. ${ }^{19}$

\section{B On the one-point function in the ZZ and AdS formulations}

Let us make few comments on the tadpole contributions in the ZZ and AdS formulations discussed in section 2.1. As follows from (2.17), the tadpole $\langle\chi(z)\rangle^{Z Z}$ has a non-constant $\mathcal{O}(b)$ contribution that combined with the classical background (2.2) changes its coefficient from $b^{-1}$ to $Q=b^{-1}+b$. The constant part of the $\mathcal{O}(b)$ term in (2.16), (2.17) is regularization dependent.

In general, the two-loop purely tadpole corrections to the one-point function are given by

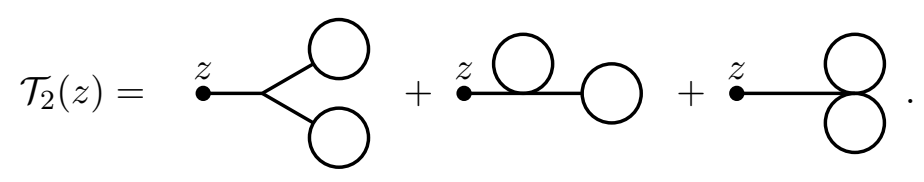

These are absent in the AdS formulation (where all tadpoles vanish, see (2.14)) but are non-zero and constant in the ZZ formulation. While separate contributions to (B.1) are not $\mathrm{SU}(1,1)$ covariant, their sum is and it may be written as (see also [32])

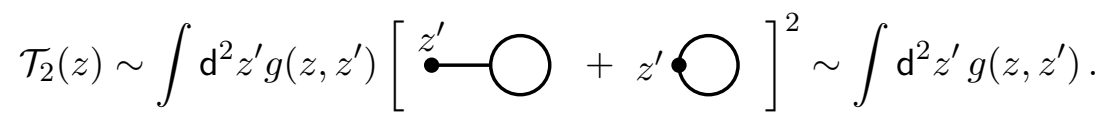

This is just a $z$-independent constant (as one can see, e.g., by using $\mathrm{SU}(1,1)$ invariance or comparing (A.5) and (A.7)). In a general regularization scheme in (2.12) one thus finds that $\mathcal{T}_{2}(z) \sim \mathrm{q}_{1}^{2}$ (cf. (A.8)). This is consistent with the vanishing of $\mathcal{T}_{2}(z)$ in the Hadamard-like regularization (where $\mathrm{q}_{1}=0, \mathrm{q}_{2}=$ const in (2.12)) discussed in [18].

\section{Tree level Witten diagram contributions to $\left\langle\Phi\left(t_{1}\right) \cdots \Phi\left(t_{4}\right)\right\rangle$}

At the tree level, the connected four-point boundary correlator $\mathrm{C}\left(t_{1}, \ldots, t_{4}\right) \equiv$ $\left\langle\Phi\left(t_{1}\right) \cdots \Phi\left(t_{4}\right)\right\rangle_{\text {conn }}$ is given by the sum of the contact and the exchange diagrams (cf.

\footnotetext{
${ }^{18}$ For instance, the diagram associated with $D\left(z_{1}, z_{2}\right)$ must be multiplied by $(-8)^{2}$ from the cubic couplings and by the symmetry factor $\frac{1}{2}$ giving +32 in (A.17).

${ }^{19}$ Note also that $\widehat{\mathrm{B}}\left(z_{1}, z_{2}\right)$ coincides with the expression in eq. (86) of [18] because the r.h.s. of that equation is given by the diagrams associated with $\widehat{\mathrm{B}}\left(z_{1}, z_{2}\right)$.
} 
$C_{4,0}^{\text {cont }}$ and $C_{4,0}^{\text {exch }}$ in (4.4)). Their separate contributions is straightforward to compute [19]:

$$
\text { (C.1) }
$$

where the function $D_{n, k, l, m}\left(t_{1}, \ldots, t_{4}\right)$ are the standard AdS integrals defined in [33-35]. Their explicit evaluation gives

$$
\begin{aligned}
& \mathrm{C}_{4,0}^{\mathrm{cont}}=\frac{64}{81 t_{12}^{4} t_{34}^{4}}\left[\frac{2\left(\chi^{2}-\chi+1\right) \chi^{2}}{(1-\chi)^{2}}+\left(2 \chi^{2}+\chi+2\right) \chi \log (1-\chi)+\frac{\left(2 \chi^{2}-5 \chi+5\right) \chi^{4} \log \chi}{(1-\chi)^{3}}\right], \\
& \mathrm{C}_{4,0}^{\mathrm{exch}}=\frac{64}{81 t_{12}^{4} t_{34}^{4}}\left[\frac{\left(\chi^{2}-\chi+1\right) \chi^{2}}{(1-\chi)^{2}}-\left(2 \chi^{2}+\chi+2\right) \chi \log (1-\chi)\right. \\
& \left.-\frac{\left(2 \chi^{2}-5 \chi+5\right) \chi^{4} \log \chi}{(1-\chi)^{3}}\right]
\end{aligned}
$$

where $\chi=\frac{t_{12} t_{34}}{t_{13} t_{24}}$ is the $1 \mathrm{~d}$ cross-ratio. Note that these two contributions have non-trivial logarithmic dependence on the boundary points $t_{1}, \ldots, t_{4}$. However, all the logarithms cancel in their sum which becomes simply proportional to the conformal factor $\mathcal{K}_{4}$ in (4.7):

$$
\begin{aligned}
& \mathrm{C}_{4,0}^{\mathrm{cont}}\left(t_{1}, \ldots, t_{4}\right)+\mathrm{C}_{4,0}^{\mathrm{exch}}\left(t_{1}, \ldots, t_{4}\right) \\
& \quad=\frac{64}{27} \frac{1}{t_{12}^{4} t_{34}^{4}} \frac{\chi^{2}\left(1-\chi+\chi^{2}\right)}{(1-\chi)^{2}}=\frac{32}{27}\left(\frac{1}{t_{12}^{2} t_{23}^{2} t_{34}^{2} t_{14}^{2}}+\frac{1}{t_{13}^{2} t_{24}^{2} t_{14}^{2} t_{23}^{2}}+\frac{1}{t_{12}^{2} t_{24}^{2} t_{34}^{2} t_{13}^{2}}\right)
\end{aligned}
$$

reproducing the value for the coefficient $C_{4,0}$ in (4.4).

Note that this cancellation does not happen if the two contributions are combined with different weights like in (4.6) where $C_{4,0}^{\text {cont }}$ and $C_{4,0}^{\text {exch }}$ should then be understood as the functions in (C.2) divided by the factor $\mathcal{K}_{4}$.

\section{Global conformal block decomposition of the four-point correlator}

The four-point correlator in (1.11)-(1.13) has a simple dependence on the boundary points without any logarithmic terms of the $1 \mathrm{~d}$ cross ratio $\chi$. This means that the $1 \mathrm{~d}$ conformal operators appearing in the OPE of the boundary correlator (1.11) will have no anomalous dimensions (cf. $[12,14]$ ). The same structure is fixed by the Virasoro symmetry in the case of the stress tensor correlator (1.5). In view of this connection it is of some interest to discuss the equivalent OPE decomposition of the stress tensor correlator in terms of the conformal blocks of the global part of the $2 \mathrm{~d}$ conformal symmetry (which is effectively equivalent to $1 \mathrm{~d}$ conformal block decomposition). 
One can represent the correlator in (1.5) as

$$
\begin{aligned}
& \left\langle T\left(z_{1}\right) T\left(z_{2}\right) T\left(z_{3}\right) T\left(z_{4}\right)\right\rangle=\frac{1}{z_{12}^{4} z_{34}^{2}} G(\chi), \quad \chi \equiv \frac{z_{12} z_{34}}{z_{13} z_{24}}, \\
& G(\chi)=\frac{c^{2}}{4}\left[1+\chi^{4}+\frac{\chi^{4}}{(1-\chi)^{4}}\right]+2 c \frac{\chi^{2}\left(1-\chi+\chi^{2}\right)}{(1-\chi)^{2}}
\end{aligned}
$$

where the $\frac{c^{2}}{4}$ term is the "generalized free field" part. From the global $\operatorname{SL}(2, \mathbb{R})$ invariance we should have

$$
G(\chi)=\sum_{n=0}^{\infty} c_{n} \mathrm{~F}_{n}(\chi), \quad \mathrm{F}_{n}(\chi)=\chi_{2}^{n} F_{1}(n, n, 2 n ; \chi), \quad c_{n}=\sum_{\mathcal{O}_{n}} \frac{\mathrm{C}_{3, \mathcal{O}_{n}}^{2}}{\mathrm{C}_{2, \mathcal{O}_{n}}},
$$

where $\left\{\mathcal{O}_{n}\right\}$ stand for the dimension $n$ quasi-primary fields (with $T$ corresponding to $\left\{\mathcal{O}_{2}\right\}$ ) in the vacuum module and $\mathrm{C}_{3, \mathcal{O}}$ and $\mathrm{C}_{2, \mathcal{O}}$ are the coefficients in $\langle T T \mathcal{O}\rangle$ and $\langle\mathcal{O O}\rangle$. One can show by a direct analysis of the algebraic expression in (D.2) that [36]

$c_{2 p+2}=\left[\frac{c^{2}}{144}(2 p-1)_{6}+2 c[1+2 p(2 p+3)]\right] \frac{(2 p) !(2 p+1) !}{(4 p+1) !}, \quad c_{2 p+1}=0, \quad p=0,1,2, \ldots$.

Thus

$$
G(\chi)=\frac{c^{2}}{4}+2 c \mathrm{~F}_{2}(\chi)+\left(\frac{c^{2}}{2}+\frac{11}{5} c\right) \mathrm{F}_{4}(\chi)+\left(\frac{10}{9} c^{2}+\frac{29}{63} c\right) \mathrm{F}_{6}(\chi)+\mathcal{O}\left(\chi^{8}\right) .
$$

Here $c_{0}=\frac{c^{2}}{4}$ and $c_{2}=2 c$ are associated with $\mathcal{O}_{0}=\mathbb{I}$ and $\mathcal{O}_{2}=T$. At level 4 we have the quasi-primary field (appearing in the regular part of the OPE of $T(z) T\left(z^{\prime}\right)$ )

$$
\Lambda_{4}=(T T)-\frac{3}{10} T^{\prime \prime}
$$

where the brackets denote the normal ordering. Using that $\mathrm{C}_{2, \Lambda_{4}}=\mathrm{C}_{3, \Lambda_{4}}=\frac{c(22+5 c)}{10}$, we confirm the value of $c_{4}$ in (D.5). At level 6 one finds two orthogonal quasi-primaries

$$
\begin{aligned}
\Lambda_{6}^{(a)}= & (T(T T))-\frac{9}{8}\left(T^{\prime} T^{\prime}\right)-\frac{1}{112} T^{\prime \prime \prime \prime} \\
\Lambda_{6}^{(b)}= & (T(T T))-\frac{28 c^{2}+1050 c+2735}{18(42 c+67)}\left(T^{\prime \prime} T\right) \\
& +\frac{35 c^{2}+462 c+2062}{18(42 c+67)}\left(T^{\prime} T^{\prime}\right)-\frac{5 c^{2}+228 c+553}{108(42 c+67)} T^{\prime \prime \prime \prime},
\end{aligned}
$$

and a straightforward calculation gives

$$
\begin{aligned}
\mathrm{C}_{2, \Lambda_{6}^{(a)}} & =\frac{3}{112} c\left(28 c^{2}+1050 c+2735\right), \\
\mathrm{C}_{2, \Lambda_{6}^{(b)}} & =\frac{c(2 c-1)(5 c+22)(7 c+68)\left(28 c^{2}+1050 c+2735\right)}{36(42 c+67)^{2}} \\
\mathrm{C}_{3, \Lambda_{6}^{(b)}} & =-\frac{c(2 c-1)(5 c+22)(7 c+68)}{9(42 c+67)} .
\end{aligned}
$$

As a result, $c_{6}$ in (D.3) is given by the sum

$$
c_{6}=\frac{3 c(42 c+67)^{2}}{7\left(28 c^{2}+1050 c+2735\right)}+\frac{4 c(2 c-1)(5 c+22)(7 c+68)}{9\left(28 c^{2}+1050 c+2735\right)}=\frac{10}{9} c^{2}+\frac{29}{63} c,
$$

in agreement with (D.5). 
Open Access. This article is distributed under the terms of the Creative Commons Attribution License (CC-BY 4.0), which permits any use, distribution and reproduction in any medium, provided the original author(s) and source are credited.

\section{References}

[1] E. D'Hoker and R. Jackiw, Space translation breaking and compactification in the Liouville theory, Phys. Rev. Lett. 50 (1983) 1719 [INSPIRE].

[2] E. D'Hoker, D.Z. Freedman and R. Jackiw, $\mathrm{SO}(2,1)$ invariant quantization of the Liouville theory, Phys. Rev. D 28 (1983) 2583 [InSPIRE].

[3] T. Inami and H. Ooguri, Dynamical breakdown of supersymmetry in two-dimensional anti-de Sitter space, Nucl. Phys. B 273 (1986) 487 [InSPIRE].

[4] C.G. Callan Jr. and F. Wilczek, Infrared behavior at negative curvature, Nucl. Phys. B 340 (1990) 366 [INSPIRE].

[5] A.B. Zamolodchikov and A.B. Zamolodchikov, Liouville field theory on a pseudosphere, hep-th/0101152 [InSPIRE].

[6] D. Carmi, L. Di Pietro and S. Komatsu, A study of quantum field theories in AdS at finite coupling, JHEP 01 (2019) 200 [arXiv:1810.04185] [INSPIRE].

[7] N. Drukker, D.J. Gross and A.A. Tseytlin, Green-Schwarz string in $A d S_{5} \times S^{5}$ : semiclassical partition function, JHEP 04 (2000) 021 [hep-th/0001204] [INSPIRE].

[8] L.F. Alday and J. Maldacena, Comments on gluon scattering amplitudes via AdS/CFT, JHEP 11 (2007) 068 [arXiv:0710.1060] [INSPIRE].

[9] A.M. Polyakov and V.S. Rychkov, Gauge field strings duality and the loop equation, Nucl. Phys. B 581 (2000) 116 [hep-th/0002106] [INSPIRE].

[10] J. Polchinski and J. Sully, Wilson loop renormalization group flows, JHEP 10 (2011) 059 [arXiv: 1104.5077] [INSPIRE].

[11] N. Drukker and S. Kawamoto, Small deformations of supersymmetric Wilson loops and open spin-chains, JHEP 07 (2006) 024 [hep-th/0604124] [INSPIRE].

[12] S. Giombi, R. Roiban and A.A. Tseytlin, Half-BPS Wilson loop and $A d S_{2} / C F T_{1}$, Nucl. Phys. B 922 (2017) 499 [arXiv:1706.00756] [INSPIRE].

[13] M. Beccaria and A.A. Tseytlin, On non-supersymmetric generalizations of the Wilson-Maldacena loops in $N=4$ SYM, Nucl. Phys. B 934 (2018) 466 [arXiv:1804.02179] [INSPIRE].

[14] M. Beccaria, S. Giombi and A.A. Tseytlin, Correlators on non-supersymmetric Wilson line in $N=4 S Y M$ and $A d S_{2} / C F T_{1}$, JHEP 05 (2019) 122 [arXiv: 1903.04365] [inSPIRE].

[15] A.M. Polyakov, Quantum geometry of bosonic strings, Phys. Lett. B 103 (1981) 207 [INSPIRE].

[16] J. Teschner, Liouville theory revisited, Class. Quant. Grav. 18 (2001) R153 [hep-th/0104158] [INSPIRE].

[17] Y. Nakayama, Liouville field theory: a decade after the revolution, Int. J. Mod. Phys. A 19 (2004) 2771 [hep-th/0402009] [INSPIRE]. 
[18] P. Menotti and E. Tonni, Standard and geometric approaches to quantum Liouville theory on the pseudosphere, Nucl. Phys. B 707 (2005) 321 [hep-th/0406014] [INSPIRE].

[19] H. Ouyang, Holographic four-point functions in Toda field theories in AdS $S_{2}$, JHEP 04 (2019) 159 [arXiv: 1902.10536] [INSPIRE].

[20] A. Strominger, AdS 2 quantum gravity and string theory, JHEP 01 (1999) 007 [hep-th/9809027] [INSPIRE].

[21] A. Almheiri and J. Polchinski, Models of AdS $S_{2}$ backreaction and holography, JHEP 11 (2015) 014 [arXiv: 1402.6334] [INSPIRE].

[22] J. Maldacena, D. Stanford and Z. Yang, Conformal symmetry and its breaking in two dimensional nearly anti-de-Sitter space, PTEP 2016 (2016) 12C104 [arXiv:1606.01857] [INSPIRE].

[23] J. Engelsöy, T.G. Mertens and H. Verlinde, An investigation of $A d S_{2}$ backreaction and holography, JHEP 07 (2016) 139 [arXiv:1606.03438] [INSPIRE].

[24] J.-L. Gervais and A. Neveu, New quantum treatment of Liouville field theory, Nucl. Phys. B 224 (1983) 329 [INSPIRE].

[25] P. Mansfield, Light cone quantization of the Liouville and Toda field theories, Nucl. Phys. B 222 (1983) 419 [INSPIRE].

[26] E. Braaten, T. Curtright, G. Ghandour and C.B. Thorn, A class of conformally invariant quantum field theories, Phys. Lett. B 125 (1983) 301 [INSPIRE].

[27] C. Ahn, C. Rim and M. Stanishkov, Exact one point function of $N=1$ super-Liouville theory with boundary, Nucl. Phys. B 636 (2002) 497 [hep-th/0202043] [InSPIRE].

[28] L.A. Takhtajan, Topics in quantum geometry of Riemann surfaces: two-dimensional quantum gravity, in Como quantum groups, Villa Monastero, Varenna, Italy (1994), pg. 541 [hep-th/9409088] [INSPIRE].

[29] P. Menotti and E. Tonni, The tetrahedron graph in Liouville theory on the pseudosphere, Phys. Lett. B 586 (2004) 425 [hep-th/0311234] [INSPIRE].

[30] D.Z. Freedman, S.D. Mathur, A. Matusis and L. Rastelli, Correlation functions in the $C F T_{d} / A d S_{d+1}$ correspondence, Nucl. Phys. B 546 (1999) 96 [hep-th/9804058] [INSPIRE].

[31] T. Hahn, CUBA: a library for multidimensional numerical integration, Comput. Phys. Commun. 168 (2005) 78 [hep-ph/0404043] [INSPIRE].

[32] P. Menotti and E. Tonni, Liouville field theory with heavy charges. I. The pseudosphere, JHEP 06 (2006) 020 [hep-th/0602206] [INSPIRE].

[33] E. D'Hoker, D.Z. Freedman, S.D. Mathur, A. Matusis and L. Rastelli, Graviton exchange and complete four point functions in the AdS/CFT correspondence, Nucl. Phys. B 562 (1999) 353 [hep-th/9903196] [INSPIRE].

[34] F.A. Dolan and H. Osborn, Conformal four point functions and the operator product expansion, Nucl. Phys. B 599 (2001) 459 [hep-th/0011040] [INSPIRE].

[35] F.A. Dolan and H. Osborn, Conformal partial waves and the operator product expansion, Nucl. Phys. B 678 (2004) 491 [hep-th/0309180] [INSPIRE].

[36] H. Osborn, Conformal blocks for arbitrary spins in two dimensions, Phys. Lett. B 718 (2012) 169 [arXiv:1205.1941] [INSPIRE]. 